Research report

\title{
The effects of lamotrigine and ethosuximide on seizure frequency, neuronal loss, and astrogliosis in a model of temporal-lobe epilepsy
}

\author{
Jie Wang ${ }^{\mathrm{a}}$, Yanjun Chen ${ }^{\mathrm{a}}$, Qiong Wang ${ }^{\mathrm{a}}$, Gilles van Luijtelaar ${ }^{\mathrm{b}}$, Meizhen Sun ${ }^{\mathrm{a}, *}$ \\ ${ }^{a}$ Neurology Department of the First Hospital of Shanxi Medical University, Taiyuan, Shanxi, China \\ ${ }^{\mathrm{b}}$ Department of Biological Psychology, Donders Centre for Cognition, Radboud University, Nijmegen, the Netherlands
}

\section{H I G H L I G H T S}

- Lamotrigine showed disease-modifying and neuroprotective effects in the model.

- Ethosuximide failed to have disease-modifying effects in the model.

- The reduced astrogliosis and neuronal loss were factors of disease-modifying effects.

\section{A R T I C L E I N F O}

\section{Keywords:}

Epilepsy

Lithium-pilocarpine

Lamotrigine

Ethosuximide

Neuroprotection

Disease-modifying

\begin{abstract}
A B S T R A C T
Objective: The putative neuroprotective and disease-modifying effects of lamotrigine (LTG) and ethosuximide (ESM) were investigated in the lithium-pilocarpine (Li-Pc) model of temporal-lobe epilepsy (TLE) in rats. Then, spontaneous recurrent seizures (SRS), neuronal loss and astrogliosis were assessed.

Methods: Status Epilepticus (SE) was induced by Li-Pc in five experimental groups: $24 \mathrm{~h}$ after SE, all rats received twice daily either a low $(10 \mathrm{mg} / \mathrm{kg})$ or high $(20 \mathrm{mg} / \mathrm{kg})$ dose of LTG, or a low $(25 \mathrm{mg} / \mathrm{kg}) \mathrm{or} \mathrm{high}(50 \mathrm{mg} / \mathrm{kg})$ dose of ESM, or solvent. The sixth group (control) did not receive Li-Pc and was given twice daily injections with solvent only. Drug administration lasted for $7 \mathrm{~d}$. Rats were systematically observed in the 5th and 6th weeks, after that the brains were prepared for histology.

Results: LTG dose-dependently decreased the frequency of SRS, and restricted neuronal loss, as well as astrogliosis in the hippocampus compared with the untreated SE control group. However, ESM had none of the abovementioned effects.

Conclusion: LTG had protective as well as disease-modifying effects in this TLE model. It was revealed that the disease-modifying effects were accompanied by the prevention of neuronal loss and astrogliosis. ESM was devoid of antiepileptogenic and its accompanying histological effects in this TLE model, in contrast to the antiepileptogenic effects found in the genetic absence epilepsy models, suggesting that different mechanisms are involved in the different models for epileptogenesis and antiepileptogenesis.
\end{abstract}

\section{Introduction}

Temporal-lobe epilepsy (TLE) is one of the most drug-resistant types of epilepsy. Thus, this demands other therapeutic approaches than the classical treatment with ant-epileptic drugs; one of which is the prophylactic treatment aiming at preventing epileptogenesis or disease modification (Schmidt and Löscher, 2005; Dichter, 2009). Various animal models and methods have been used to find out disease modifying medication in TLE, such as electrical or chemical hippocampal or amygdala kindling (Stratton et al., 2003), febrile seizures (Dubé et al., 2007), and different post-status epilepticus (SE) models (Turski et al., 1983a,b). The post-SE models come close to being homologous for TLE (Morimoto et al., 2004), although some of them are also criticized because of their wide spread extra hippocampus tissue damage (Lévesque et al., 2016). Rats develop spontaneous recurrent seizures (SRS), hippocampal and extra-hippocampal damage, as well as behavioral and cognitive alterations resembling the clinical characteristics of TLE after a systemic injection with the muscarinic cholinergic agonist pilocarpine (Morimoto et al., 2004).

Older and newer anti-epileptic medications, including lamotrigine (LTG), have been investigated for their antiepileptogenic or disease modifying and neuroprotective effects in a variety of TLE models with

\footnotetext{
* Corresponding author.

E-mail address: sunmeizhen213@126.com (M. Sun).
} 
different results. LTG demonstrated antiepileptogenic-like effects by blocking seizure development during treatment period in a rat amygdala (Stratton et al., 2003) and PTZ kindling model (Chen et al., 2017). However, LTG was given in both studies prior to the daily kindling sessions and this may have curtailed the intensity and duration of the after discharge (AD); as a consequence, this might have slowed down epileptogenesis.

Curtailing rarely occurs in the post-SE models when drug administration is initiated after SE onset, or better after SE offset. For example, Nissinen et al. (2004) attempted to start LTG treatment $2 \mathrm{~h}$ after the induction of electrically induced SE, and their treatment lasted 11 weeks. Antiepileptic effects were found, while there were no antiepileptogenic effects in this electrically induced post-SE model.

Curtailing can be fully prevented in post-SE models if treatment started when SE is completely over, for example $24 \mathrm{~h}$ after SE. Here, the effects of the putative antiepileptogenic action of LTG in the LithiumPilocarpine (Li-Pc) post-SE model, most commonly used for studying epileptogenesis, were evaluated in rats. In an earlier study performed by using this model, seizures were counted and histology was carried out during LTG treatment (Chen et al., 2010) without a wash-out period, therefore, an antiepileptic action was investigated and established.

Antiepileptogenic and disease modifying effects of Ethosuximide (ESM), a Ca ${ }^{2+}$ channel blocker, have been described in a genetic type of absence epilepsy model, rats of the WAG/Rij strain. The age-dependent increase of spike-wave discharges (SWDs), upregulation of cortical $\mathrm{Na}^{+}$, and downregulation of HCN channels were diminished as a consequence of early and chronic treatment until 2-4 months after stopping its administration (Blumenfeld et al., 2008; Sarkisova et al., 2010; van Luijtelaar et al., 2013; Russo et al., 2010). There are also indications that ESM might play a major role in antiepileptogenesis in the Li-Pc post-SE model: the RNA and protein of the subtype T-type $\mathrm{Ca}^{2+}$ channel, Cav3.2 was upregulated and caused an increase in T-type $\mathrm{Ca}^{2+}$ currents, in addition to a transitional increase in intrinsic burst firing in mice (Becker et al., 2008). In the present study, we aimed to find out whether LTG and ESM have neuroprotective and antiepileptogenic or disease-modifying effects in the Li-Pc post-SE model of TLE.

\section{Results}

\subsection{The effects of LTG and ESM on seizure frequency}

Within 5 min after intraperitoneal injection of Li-Pc, the rats showed a shrinking pupil reaction, salivation and diarrhea, as well as facial clonus, including eye blinks, rhythmic chewing and nodding, in which forelimb spasms appeared. About $30 \mathrm{~min}$ after SE, rats developed a rearing position or tonic-clonic seizures appeared. Here, 58 rats were used in the experiment. In addition to 8 rats in the control group, 50 rats were injected with Li-Pc. Besides, 6 of the 50 injected rats showed only seizure stages I-III without SE; 4 of the 50 rats died because of severe SE; 40 of 50 rats got SE, survived, and then were randomly divided into five groups, and each group consisted of eight rats.

After the acute phase of SE, the rats entered the static period, which showed less eating, irritability, and restlessness. 15 days after SE induction, the rats entered the chronic phase. SRS of magnitude III-V could be observed and continued for 1-2 min in the model group. The duration of seizures varied roughly between 10 and $15 \mathrm{~s}$ for rats in the LTG groups, and frequency of SRS (the number of SRS per week) was significantly and dose-dependently lower compared with the model group $(\mathrm{P}<0.05)$. The frequency of SRS for both ESM groups did not significantly decrease compared with the model group (See Fig. 1, $P>0.05)$.

\subsection{The effects of LTG and ESM on hippocampal neurons}

The CA3 area contained several Nissl stained neurons (See Figs. 2

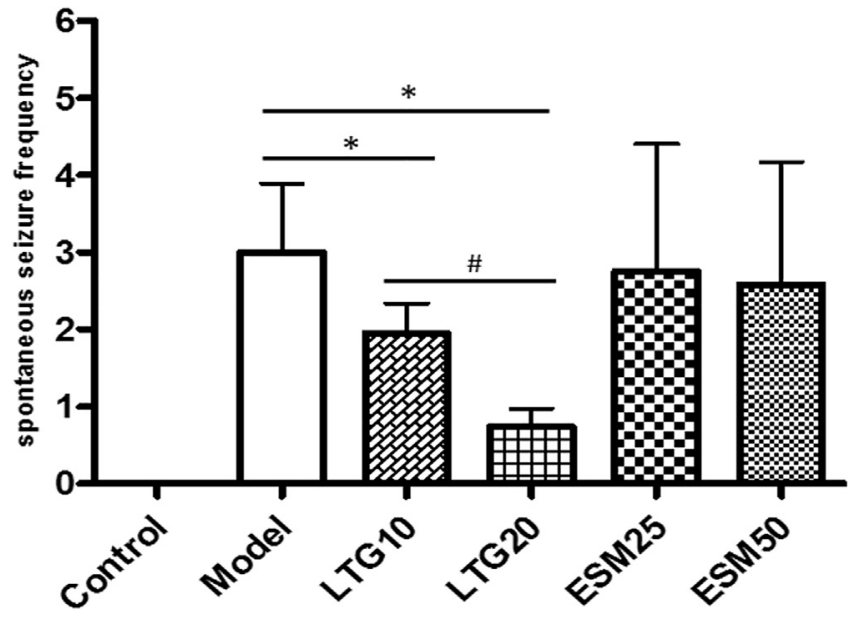

Fig. 1. The effects of LTG and ESM on the number of SRS per week ( $n=8$ per group), as observed during 5 and 6 weeks after SE. LTG decreased the frequency of SRS in a dose-dependent manner, while ESM had no significant effect. ${ }^{*} \mathrm{P}<0.05$ compared with the model group; \#P $<0.05$ compared with the low dose LTG group. Y-axis: the number of SRS per week, mean and standard deviation (SD) were plotted.
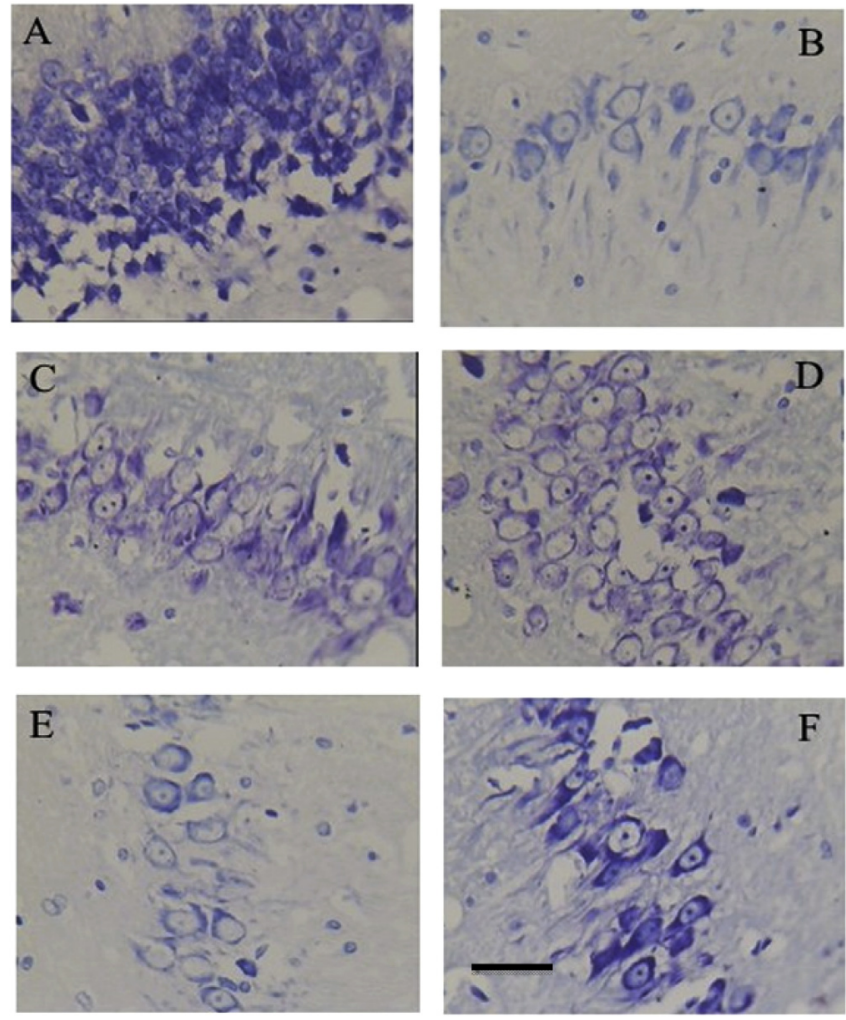

Fig. 2. Hippocampal neurons in CA3 area in different groups ( $n=8$ per group), in which mean and SD were plotted. A: Normal control group; B: Model group; C: LTG group (10 mg/kg, twice daily); D: LTG group (20 mg/kg, twice daily); E: ESM group ( $25 \mathrm{mg} / \mathrm{kg}$, twice daily); F: ESM group $(50 \mathrm{mg} / \mathrm{kg}$, twice daily). Coronal brain paraffin sections (thickness $=4 \mu \mathrm{m}$ ) were examined using light microscopy ( $\times 400$ magnification). In the dorsal of CA3 area, five non-overlapping visual fields were randomly sampled to count the number of positive Nissl-stained neurons in each field $\left(625 \mu \mathrm{m}^{2}\right)$. Scale bar (A, B, C, D, E, F) is $100 \mu \mathrm{m}$.

and 3). The nuclei were dyed blue and the Nissl stained cell bodies were abundantly present. The Nissl bodies presented themselves in patches or in fine granular layers. In the control group, the neurons were packed 


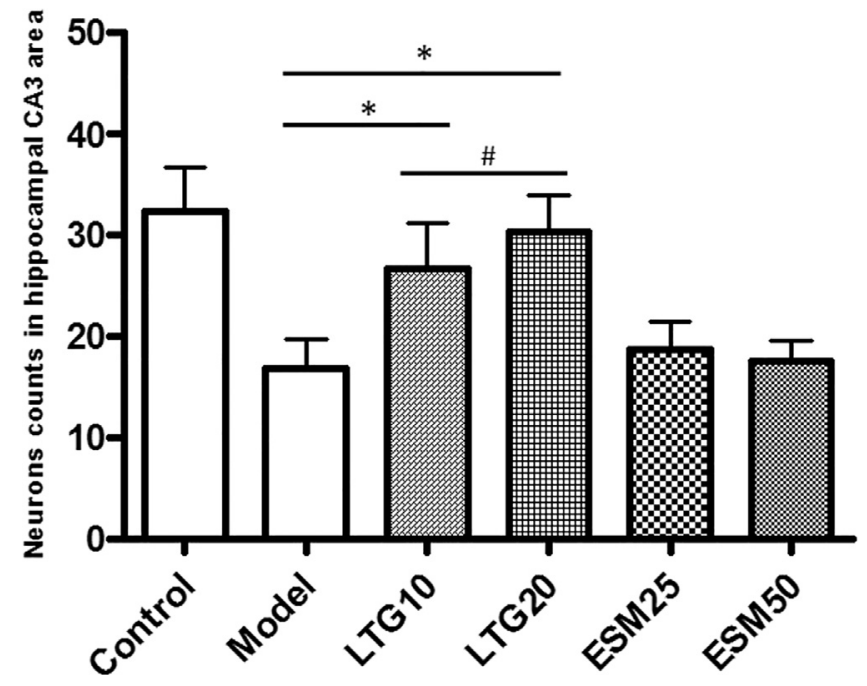

Fig. 3. The effects of LTG and ESM on the number of neurons counted in the hippocampal CA3 area ( $n=8$ per group), in which mean and SD were plotted. The number of Nissl stained neurons was significantly lower in the model group compared with the control group. The neuronal loss remained dose-dependently restricted by the intervention with lamotrigine (LTG10 and LTG20, twice daily), while the ethosuximide (ESM25 and ESM50, twice daily) treated rats did not differ from the model group. ${ }^{*} \mathrm{P}<0.05$ compared with the model group; \#P $<0.05$ compared with the lower dose LTG group.

closely and the edges were clear. In the model group, the neurons were diffusely and sparsely arranged. Dying cells were identified: the size of neurons became smaller with a wrinkled cell membrane; the nuclei were more condense, or the nuclei had disappeared and only a trace of the membrane of the nuclei was present. The quantification revealed that the number of neurons in the model group was fewer compared with that in the control group $(\mathrm{P}<0.05)$. The neuronal loss and degree of damage were reduced significantly and dose-dependently in the LTG groups compared with the model group (all $\mathrm{P}<0.05$ ). However, no significant differences were found in the groups receiving ESM compared with the model group or between the ESM groups $(\mathrm{P}>0.05)$.

\subsection{The effects of LTG and ESM on hippocampal astrogliosis}

Glial fibrillary acidic protein (GFAP) staining was performed in order to evaluate astrogliosis in the CA1 area of the hippocampus. The number of immune reaction positive cells was fewer $(\mathrm{P}<0.05)$ in the control group compared with the other groups (except in the higher dose LTG group). A qualitative impression of the hippocampal astrocytes is given in Fig. 4. The number of immune reaction positive astrocytes increased in the model group, and the size of the astrocytes became larger with thicker cell membrane and shorter dendrite branch. Astrogliosis was remarkably and dose-dependently reduced in the LTG groups compared with the model group $(\mathrm{P}<0.05)$. However, there were no significant differences between the rats that were treated with ESM of between the ESM groups and the model group ( $P>0.05$, see Fig. 5).

\section{Discussion}

The Post-SE Li-Pc model was used to study the effects of two antiepileptic drugs on epileptogenesis in TLE. Treatment with two doses of LTG (20 and $40 \mathrm{mg} / \mathrm{kg} /$ day) for $7 \mathrm{~d}$, initiated that $1 \mathrm{~d}$ after SE, the number of SRS was reduced dose-dependently. Considering that LTG was only first administered $24 \mathrm{~h}$ after SE and the number of SRS was counted after complete LTG wash-out, our design precluded putative anti-epileptic effects of the medication. Therefore, it can be concluded
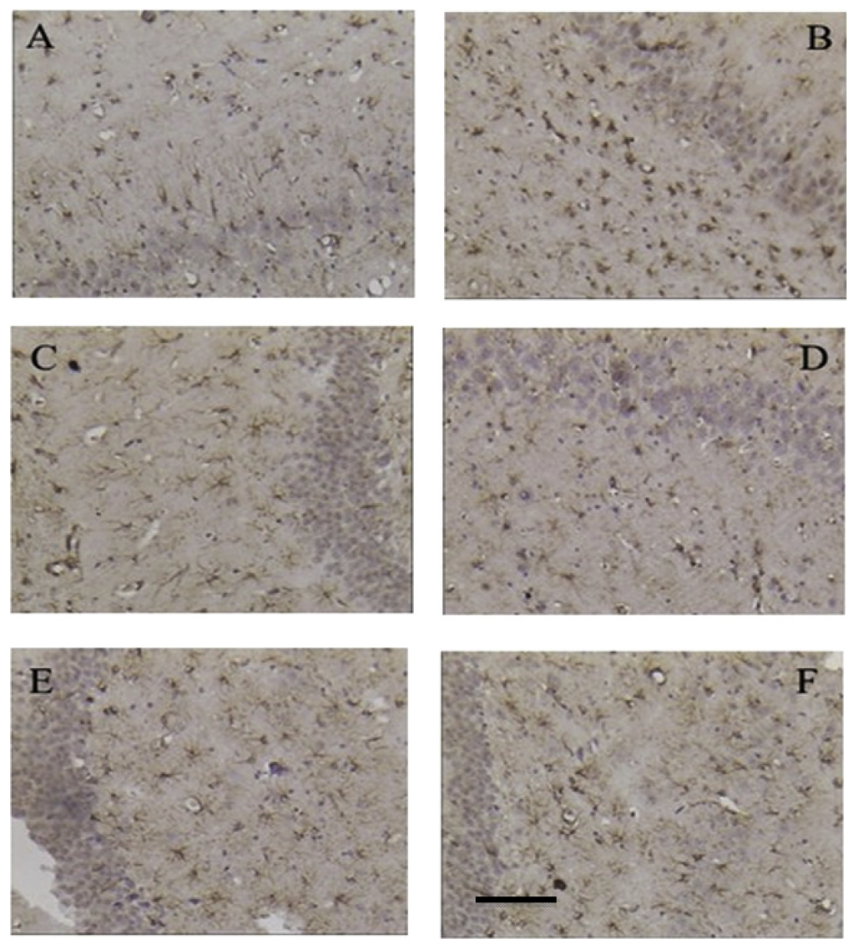

Fig. 4. Hippocampal astrogliosis in the CA1 area in different groups $(n=8$ per group). A: Normal control group; B: Model group; C: LTG group $(10 \mathrm{mg} / \mathrm{kg}$, twice daily); D: LTG group (20 mg/kg, twice daily); E: ESM group $(25 \mathrm{mg} / \mathrm{kg}$, twice daily); F: ESM group $(50 \mathrm{mg} / \mathrm{kg}$, twice daily). Coronal brain paraffin sections (thickness $=4 \mu \mathrm{m})$ were examined using light microscopy $(\times 200$ magnification). In the dorsal of CA1 area, five non-overlapping visual fields were randomly sampled to count positive GFAP-staining astrocytes in each field $\left(1250 \mu \mathrm{m}^{2}\right)$. Scale bar (A, B, C, D, E, F): $200 \mu \mathrm{m}$. Mean numbers and SD were plotted.

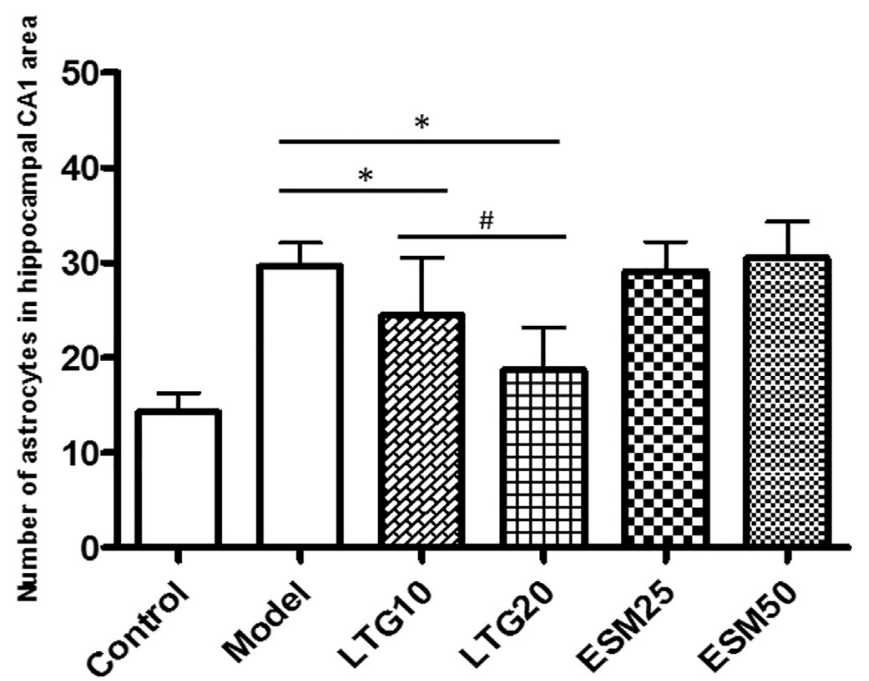

Fig. 5. The effects of LTG and ESM on the number of astrocytes in the CA1 area of the hippocampus ( $n=8$ per group). The number of astrocytes (mean and SD) was significantly increased in the model group compared with the control group (not indicated), and this effect was reversed by lamotrigine (LTG10 and LTG20, twice daily) intervention, while ethosuximide (ESM25 and ESM50, twice daily) had no significant effect. ${ }^{*} \mathrm{P}<0.05$ compared with the model group; $\# \mathrm{P}<0.05$ indicates that there is a significant difference between LTG10 and LTG20 groups. 
that the outcome indicated disease-modification of LTG in this post-SE model of TLE. Disease-modifying effects were obtained (Löscher and Brandt, 2010; Giblin and Blumenfeld, 2010), not complete antiepileptogenesis, by this prophylactic treatment in the Li-Pc post-SE model since a reduction in the number of SRS was found, without a complete abolishment. Disease modification means decreasing the seizure frequency, and/or seizure intensity and/or increasing the latency for the occurrence of SRS after prophylactic treatment.

Our results with LTG are difficult to be compared with those achieved by kindling models of TLE, and in some studies (Stratton et al., 2003; Chen et al., 2017), antiepileptogenesis or disease modifying effects were suggested. However, in the kindling models, LTG has been administered immediately prior to the electrical or chemical kindling sessions (Stratton et al., 2003; Postma et al., 2000; Chen et al., 2017), therefore, antiepileptic effects were evaluated. Sometimes antiepileptogenic effects were suggested based on the results obtained after a wash-out period, however, they might be fully or partly explained by an antiepileptic action since the intensity of the kindled seizures might be less (curtailing) due to the administration of LTG prior to kindling; consequently, epileptogenesis might be slower. Therefore, a mix of antiepileptic and antiepileptogenic effects can be suggested to explain the reduction of kindled seizures. Complete negative effects regarding antiepileptogenesis of LTG were achieved in a cortical kindling seizure model in rats (O'Donnel and Miller, 1991), suggesting that different mechanisms are involved in different models for epileptogenesis and antiepileptogenesis.

A single study towards antiepileptogenesis and disease modification used a post-SE model; in this case, SE was elicited by electrical stimulation of the amygdala (Nissinen et al., 2004). However, those authors could not demonstrate whether LTG had disease modifying or antiepileptogenic effects. The chronic LTG treatment was initiated $2 \mathrm{~h}$ after the induction of SE since the mean frequency of seizures, seizure duration, and seizure intensity did not significantly differ between the LTG and vehicle groups.

There are a few reasons, justifying different effects of LTG on antiepileptogenesis or disease-modifying in different experiments. Firstly, different models were used in these experiments, including kindling models induced by electrical stimulation of the amygdala or cortex, chemical kindling models by PTZ, post-SE model induced by electrical stimulation of the amygdala, and post-SE model induced by Li-Pc. Secondly, kindling and post-SE models also differed regarding when the drugs were administered; in the kindling models, drugs were given prior to the daily kindling sessions, while in the SE models drugs were often given immediately after the end of SE or during the silent period. Despite these differences in the two post-SE models (amygdala stimulation and Li-Pc model), it remains an open question why disease modification was only obtained in the pilocarpine model and not in the amygdala model.

LTG dose-dependently reduced neuronal loss in the CA3 area of the hippocampus compared with the model group. We selected the CA3 area based on Liu et al. study (1994) who reported a cell loss in the Pilocarpine post-SE model in rats, and they also found cell loss in the CA1 and CA 3 areas. In addition, others reported cell loss in the post-SE models in the CA3 area (Blümcke et al., 2012; Sendrowski and Sobaniec, 2013). Our data indicated that LTG had a neuroprotective function, which is also consistent with other researches' outcomes (Jung et al., 2006). In the latter study, celecoxib, a selective cyclooxygenase-2 inhibitor, attenuated the development of SRS after SE induced by pilocarpine, and also prevented neuronal death and microglia activation in the CA1 and hilus, suggesting neuroprotection. A previous study emphasized that especially neurons in the hippocampal hilus are vulnerable for seizures (Buckmaster and Jongen-Rêlo, 1999). However, neuroprotection is not always accompanied by antiepileptogenesis or disease-modification. Halonen et al. (2001) showed that LTG has neuroprotective effect, without any effect on the number or duration of behavioral seizures. In addition, Maj et al. (1998) reported that pre- treatment of rats with LTG before kainate administration could prevent hippocampal cell loss, while could not prevent seizures. It indicated that neuronal loss of hippocampal cells is only one kind of structural change, accompanying by epileptogenesis or disease-modification, however, it does not necessarily imply that the structural changes in the hippocampus necessarily cause seizures to be occurred.

LTG dose-dependently decreased astrogliosis induced by the application of our SE protocol. Astrocytic dysfunction may also play a significant role in epileptogenesis by reducing the clearance of extracellular potassium underlying neuronal hyperexcitability and network synchronization (Yaron et al., 2009). Whole-cell patch-clamp recordings from astrocytes further suggested that astrogliosis in a mouse model of genetically induced, widespread chronic astrogliosis causes an impaired astrocytic glutamate uptake, which is in parallel with heightened excitability and the occurrence of seizures (Robel et al., 2015).

Whether neuronal death and astrogliosis are the key elements in epileptogenesis should be further elucidated. A number of previous studies (e.g., Halonen et al., 2001) have shown that these features do not play a major role in epileptogenesis. Moreover, experiments conducted with N-methyl-D-aspartate (NMDA) receptor antagonists in a rat kainate-induced SE model showed that a single dose of the NMDA receptor blocker dizocilpine (MK801) given 90 min after SE resulted in hippocampal neuroprotection, while it did not prevent the development of spontaneous seizures, suggesting that structures not protected by dizocilpine may play a major role in the genesis of these seizures, or that epileptogenesis is not the consequence of structural lesions in the limbic system (Brandt et al., 2003). On the other hand, it cannot be excluded that the combined prevention of hippocampal neuronal death, astrogliosis and increased mossy fiber sprouting and most likely some other factors may restrict the chance that spontaneous seizures start to emerge.

$\mathrm{Ca}^{2+}$ ions and $\mathrm{Ca}^{2+}$ channels might play a role in epileptogenesis; preclinical data identified T-type $\mathrm{Ca}^{2+}$ channel Cav3.2 as a central player in epileptogenesis in the mice Li-Pc post-SE model, and SE caused a transient transcriptional upregulation of Cav3.2 channels (Becker et al., 2008), producing an increase in $\mathrm{T}$-type $\mathrm{Ca}^{2+}$ currents. Importantly, ESM is known to block T-type $\mathrm{Ca}^{2+}$ currents (Gomora et al., 2001), and it has disease modifying properties, as was previously shown in a genetic epilepsy model (Russo et al., 2010). However, ESM failed to protect rats against epileptogenesis, and there were neither signs of disease modification, nor signs of neuroprotection in our experiment. The highest dose of ESM was $100 \mathrm{mg} / \mathrm{kg} /$ day, divided over two injections of $50 \mathrm{mg} / \mathrm{kg} / \mathrm{per}$ day. Russo et al. (2010) mentioned that $80 \mathrm{mg} /$ day/kg ESM might already yield antiepileptogenesis in WAG/Rij rats, however, the better antiepileptogenic effects were found with $300 \mathrm{mg} / \mathrm{kg} /$ day. Therefore, the lowest dose $(50 \mathrm{mg} / \mathrm{kg} /$ day $)$ might have been very low, and the double dose might not be yielded antiepileptogenic effects. On the other hand, the duration of the twice-daily period of ESM administration might have be very short, although that would be long enough in the case of LTG; another issue is whether the mechanisms of antiepileptogenesis in absence epilepsy are identical to the mechanisms in epileptogenesis in the Li-Pc post-SE model. ESM is known not to be effective as an antiepileptic drug in models other than the absence models, and it currently seems that it lacks antiepileptogenic or disease-modifying effects and does not have a neuroprotective action in this post-SE model of TLE when administered $24 \mathrm{~h}$ after SE. These results extend the results achieved by Leite and Cavalheiro (1995), using the Li-Pc model. They administered a high ( $400 \mathrm{mg} / \mathrm{kg}$, i.p.) dose of ESM twice daily for 2 weeks during the SRS period, and found that it might be inactive against these seizures.

There are a number of limitations in our study. Firstly, we did not determine the blood levels of drugs during administration. Secondly, no electroencephalography (EEG) was carried out to identify epileptic activity during SE and the period of the SRS. 
Prophylactic treatment with LTG or ESM after SE

Experiment protocol

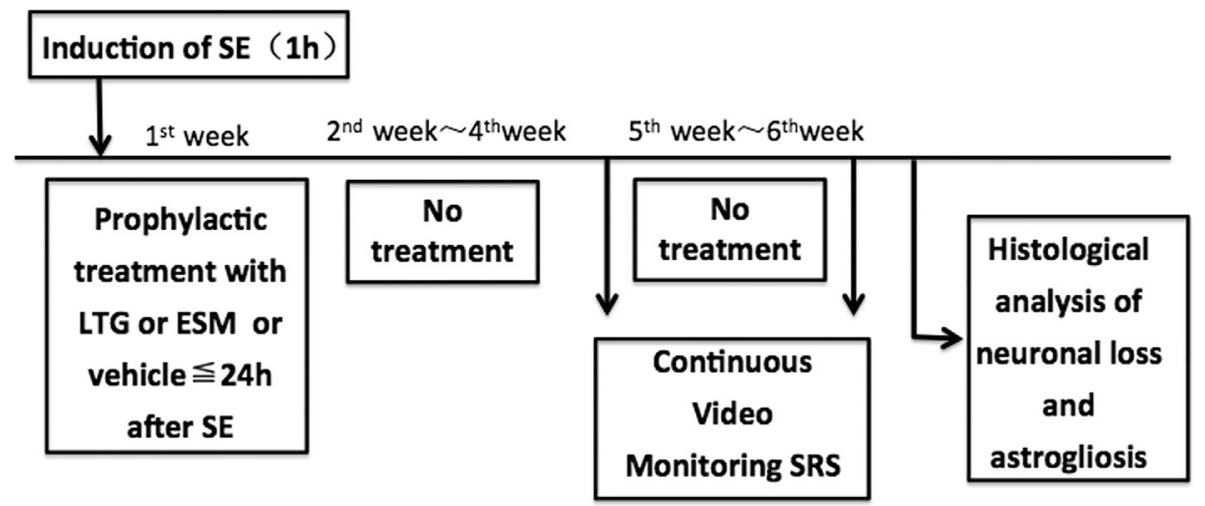

Fig. 6. Schematic illustration of the experimental protocol used in the study.

\section{Conclusions}

LTG treatment has both neuroprotective and disease-modifying effects in the post-SE model of TLE induced by Li-Pc. We propose that the disease-modifying effects occur if the reduction of astrogliosis and neuronal loss, but also other factors such as mossy fiber sprouting (Buckmaster, 2014), a loss of GABA-ergic neurons in the hilus (Buckmaster and Jongen-Rêlo, 1999) underlying the presence of the SRS, or dysregulation of an ion channel or neurotransmitter receptor, or alternations in signaling pathways, might be successfully prevented or repaired by treatment, although the prevention of hippocampal neuronal death does not seem a sufficient or necessary condition to prevent spontaneous seizures. ESM did not effect on any of these variables. In the future, it should be investigated whether or not LTG has diseasemodifying effect aiming to prevent neuronal loss and astrogliosis in models of traumatic brain injury, one of the most common brain insults.

\section{Experimental procedures}

\subsection{Animals and drugs}

The study was performed in female SD rats $(n=58)$, weighing $150-200 \mathrm{~g}$, which were provided by Shanxi Medical University. The rats were maintained under standard laboratory conditions with a natural light-dark cycle. They were given a standard pellet diet and tap water ad libitum. LTG was purchased from Glaxo Operations UK Ltd and pilocarpine from Sigma Chemical Co. (St. Louis, MO, USA). ESM was a gift from Katwijk Chemie B.V., the Netherlands. Animal welfare procedures were in accordance with common published guidelines and no more animals were used than necessary.

\subsection{Lithium Chloride-Pilocarpine epilepsy rat model}

Rats were pretreated with lithium chloride $(127 \mathrm{mg} / \mathrm{kg}$, i.p., Sigma, St. Louis, MO) and methylscopolamine-bromide ( $1 \mathrm{mg} / \mathrm{kg}$, i.p., Sigma) $18 \mathrm{~h}$ and $30 \mathrm{~min}$ before pilocarpine administration, respectively. Pilocarpine hydrochloride $(30 \mathrm{mg} / \mathrm{kg}$, i.p., Sigma) was injected to trigger SE. Repeated doses of pilocarpine hydrochloride $10 \mathrm{mg} / \mathrm{kg}$ were then administered every 30 min until stage 4 seizures developed according to Racine's scale (Racine, 1972). The dose of pilocarpine hydrochloride never exceeded $60 \mathrm{mg} / \mathrm{kg}$. Diazepam $(10 \mathrm{mg} / \mathrm{kg}$, i.p. Samjin, Seoul, Korea) was injected $1 \mathrm{~h}$ after SE in order to terminate seizure activity: its administration was repeated until SE was terminated as based on visual observations. Although it might be difficult to decide whether the animals had still had SE after the administration of diazepam, the SE rats were randomly divided across the five SE groups and at forehand there is no reason to assume that the lack of EEG control privileged any of the five conditions and that this has influenced the differences between the groups.

SRS were observed by video in all rats during the 5 th and 6 th week after SE and were scored according to Racine's scale: $0=$ no convulsive behavior; $1=$ sedation, less activity but revival of righting reflex; $2=$ facial clonus or head nodding; $3=$ forelimb clonus; $4=$ rearing, animal in a standing posture aided by tail and laterally spread hind limbs showing increased tonus; $5=$ tonic-clonic seizure.

\subsection{Study design}

Eight rats were selected randomly and served as a control group. The other rats were used for the induction of SE: after successful induction of SE, these rats were randomly divided into five groups, eight rats per group. The untreated "normal" control group was given the same volume of saline instead of Pilocarpine and Lithium chloride and for the next seven days, and it was given saline twice a day by gavage instead of LTG or ESM. The group that received solvent instead of LTG or ESM was called the "model" group. The low and high dose LTG groups were given LTG ( 10 and $20 \mathrm{mg} / \mathrm{kg}$ respectively) twice daily by gavage. The low and high dose group of ESM was given twice daily (i.p., $25 \mathrm{mg} / \mathrm{kg}$ and $50 \mathrm{mg} / \mathrm{kg}$ respectively). Both drugs were administered for the first time $24 \mathrm{~h}$ after SE and the administration was continued for seven consecutive days (See Fig. 6).

\subsection{Behavioral observations}

Here, 2 weeks after SE, the pilocarpine treated rats started to show SRS, which was called the chronic phase. Rats were monitored using a video recorder ( $24 \mathrm{~h}$ per day) from the 5 th to the end of the 6 th week after SE to evaluate the number, duration and intensity of the SRS.

\subsection{Hippocampal staining}

After the end of the observation period, all rats were anesthetized and intracardially perfused with $4 \%$ paraformaldehyde according to a fixation protocol. The fixated brain was coronally sliced for paraffin sections ( $4 \mu \mathrm{m}$ thick). Six sections came from one rat. Three paraffin sections were used for Nissl staining using standard protocols based on 
the description by Kobayashi and Buckmaster (2003). Besides, 3 paraffin sections were made for GFAP staining, using standard protocols previously described by Panagiota et al. (2010).

\subsection{Analysis of image data}

Nissl staining sections of hippocampus CA3 area were examined using light microscopy ( $\times 400$ magnification). In the dorsal section of the CA3 area, five non-overlapping visual fields were randomly sampled to count the number of positive Nissl-staining neurons in each field $\left(625 \mu \mathrm{m}^{2}\right)$. Next, the average number of Nissl-stained neurons per animal and per group (five experimental groups, one control group) was calculated.

The astrocytes stained by GFAP in CA1 region of the hippocampus were also examined using a light microscope $(\times 200$ magnification). In the dorsal of CA1 area, five non-overlapping visual fields were randomly sampled and the number of positive GFAP-staining astrocytes in each field $\left(1250 \mu \mathrm{m}^{2}\right)$ were counted. The average number of GFAPstaining astrocytes across the five fields was calculated, next the mean \pm SD of the number of labeled astrocytes were calculated for all six groups.

The counting of both Nissl stained neurons and GFAP labeled astrocytes was carried out by trained assistants using the double-blind method.

\subsection{Statistical analysis}

Data were expressed as mean \pm standard deviation (SD). The statistical analyses were performed using SPSS 17.0 software (IBM, Armonk, NY, USA). One-way analysis of variance (ANOVA) was used to intra-groups comparisons followed by Fisher's Least Significant Difference (LSD) test. $\mathrm{P}$ values $<0.05$ were considered statistically significant.

\section{Funding}

This work was supported by the International Cooperation Project, Shanxi Province, China (Grant No. 2010081069).

\section{References}

Becker, A.J., Pitsch, J., Sochivko, D., et al., 2008. Transcriptional upregulation of Cav3.2 mediates epileptogenesis in pilocarpine model of epilepsy. J. Neurosci. 28, 13341-13353.

Blümcke, I., Coras, R., Miyata, H., et al., 2012. Defining clinico-neuropathological subtypes of mesial temporal lobe epilepsy with hippocampal sclerosis. Brain Pathol. 22, 402-411.

Blumenfeld, H., Klein, J.P., Schridde, U., et al., 2008. Early treatment suppresses the development of spike-wave epilepsy in rat model. Epilepsia 49, 400-409.

Brandt, C., Potschka, H., Löscher, W., et al., 2003. N-methyl-D-aspartate receptor blockade after status epilepticus protects against limbic brain damage but not against epilepsy in the kainate model of temporal lobe epilepsy. Neuroscience 118, 727-740.

Buckmaster, P.S., 2014. Does mossy fiber sprouting give rise to the epileptic state? Adv. Exp. Med. Biol. 813, 161-168.

Buckmaster, P.S., Jongen-Rêlo, A.L., 1999. Highly specific neuron loss preserves lateral inhibitory circuits in the dentate gyrus of Kainate-Induced epileptic rats. J. Neurosci. 19, 9519-9529.

Chen, Y., He, X., Sun, Q., et al., 2017. Effect of lamotrigine on seizure development in a rat pentylenetetrazole kindling model. Brain Behav. 7, 1-10.

Chen, J., Quan, Q.Y., Yang, F., et al., 2010. Effects of lamotrigine and topiramate on hippocampal neurogenesis in experimental temporal-lobe epilepsy. Brain Res. 1313,
$270-282$.

Dichter, M.A., 2009. Posttraumatic epilepsy: the challenge of translating discoveries in the laboratory to pathways to a cure. Epilepsia 50, 41-45.

Dubé, C.M., Brewster, A.L., Richichi, C., et al., 2007. Fever, febrile seizures and epilepsy. Trends Neurosci. 30, 490-496.

Giblin, K.A., Blumenfeld, H., 2010. Is epilepsy a preventable disorder? New evidence from animal models. Neuroscientist 16, 253-275.

Gomora, J.C., Daud, A.N., Weiergräber, M., et al., 2001. Block of cloned human T-Type calcium channels by succinimide antiepileptic drugs. Mol. Pharmacol. 60, 1121-1132.

Halonen, T., Nissinen, J., Pitkanen, A., 2001. Effect of lamotrigine treatment on status epilepticus-induced neuronal damage and memory impairment in rats. Epilepsy Res. 46, 205-223.

Jung, K.H., Chu, K., Lee, S.T., et al., 2006. Cyclooxygenase-2 inhibitor, celecoxib, inhibits the altered hippocampal neurogenesis with attenuation of spontaneous recurrent seizures following pilocarpine-induced status epilepticus. Neurobiol. Dis. 23, 237-246.

Kobayashi, M., Buckmaster, P.S., 2003. Reduced inhibition of dentate granule cells in a model of temporal lobe epilepsy. J. Neurosci. 23, 2440-2452.

Leite, J.P., Cavalheiro, E.A., 1995. Effects of conventional antiepileptic drugs in a model of spontaneous recurrent seizures in rats. Epilepsy Res. 20, 93-104.

Lévesque, M., Avoli, M., Bernard, C., 2016. Animal models of temporal lobe epilepsy following systemic chemoconvulsant administration. J. Neurosci. Methods 260, 45-52.

Liu, Z., Nagao, T., Desjardins, G.C., et al., 1994. Quantitative evaluation of neuronal loss in the dorsal hippocampus in rats with long-term pilocarpine seizures. Epilepsy Res. 17, 237-247.

Löscher, W., Brandt, C., 2010. Prevention or modification of epileptogenesis after brain insults: experimental approaches and translational research. Pharmacol. Rev. 62, $668-700$.

Maj, R., Farriello, R.G., Ukmar, G., et al., 1998. PNU-151774E protects against kainateinduced status epilepticus and hippocampal lesions in the rat. Eur. J. Pharmacol. 359, 27-32.

Morimoto, K., Fahnestock, M., Racine, R.J., 2004. Kindling and status epilepticus models of epilepsy: rewiring the brain. Prog. Neurobiol. 73, 1-60.

Nissinen, J., Large, H., Stratton, S.C., et al., 2004. Effect of lamotrigine treatment on epileptogenesis: an experimental study in rats. Epilepsy Res. 58, 119-132.

O'Donnel, R.A., Miller, A.A., 1991. The effect of lamotrigine upon development of cortical kindled seizures in the rat. Neuropharmacology 30, 253-258.

Panagiota, M., Antonios, S., Fotimi, S., 2010. Neuroprotective effects of IGF-1 following kainic acid-induced hippocampal degeneration in the rat. Cell Mol. Neurobiol. 30, 347-360.

Postma, T., Krupp, E., Li, X.L., et al., 2000. Lamotrigine treatment during amygdalekindled seizure development fails to inhibit seizures and diminishes subsequent anticonvulsant efficacy. Epilepsia 41, 1514-1521.

Racine, R.J., 1972. Modification of Seizure activity by electrical stimulation II: motor seizure. Electroenceph. Clin. Neurophysiol. 32, 781-794.

Robel, S., Buckingham, S.C., Boni, J.L., et al., 2015. Reactive astrogliosis causes the development of spontaneous seizures. J. Neurosci. 35, 3330-3345.

Russo, E., Citraro, R., Scicchitano, F., et al., 2010. Comparison of the antiepileptogenic effects of an early long-term treatment with ethosuximide or levetiracetam in a genetic animal model of absence epilepsy. Epilepsia 51, 1560-1569.

Sarkisova, K.Y., Kuznetsova, G.D., Kulikov, M.A., et al., 2010. Spike-Wave discharges are necessary for the expression of behavioral depression-like symptoms. Epilepsia 51, $146-160$.

Schmidt, D., Löscher, W., 2005. Drug resistance in epilepsy: putative neurobiologic and clinical mechanisms. Epilepsia 46, 858-877.

Sendrowski, K., Sobaniec, W., 2013. Hippocampus, hippocampal sclerosis and epilepsy. Pharmacol. Rep. 65, 555-565.

Stratton, S.C., Large, C.H., Cox, B., et al., 2003. Antiepileptogenic-like effects of lamotrigine in a rat amygdale kindling model. Epilepsy Res. 53, 95-106.

Turski, W.A., Cavalheiro, E.A., Schwarz, M., et al., 1983a. Limbic seizure produced by pilocarpine in rats: behavioural, electroencephalographic and neuropathological study. Behav. Brain Res. 9, 315-335.

Turski, W.A., Czuczwar, S.J., Kleinrok, Z., et al., 1983b. Cholinomimetics produce seizures and brain damage in rats. Experientia 39, 1408-1411.

van Luijtelaar, G., Mishra, A.M., Edelbroek, P., et al., 2013. Anti-epileptogenesis: elec trophysiology, diffusion tensor imaging and behavior in a genetic absence model Neurobiol. Dis. 60, 126-138.

Yaron, D., Cacheaus, L.P., Ivens, S., et al., 2009. Astrocytic dysfunction in epileptogenesis: consequences of altered potassium and glutamate homeostasis? J. Neurosci. 29, 10588-10599. 